\title{
Forschung und Lehre zur Sozialstruktur und zu sozialer Ungleichheit an der Fakultät für Soziologie
}

\author{
Anja-Kristin Abendroth
}

Thematisch setzt sich die Lehre und Forschung im Arbeitsbereich Sozialstruktur und soziale Ungleichheit insbesondere mit der Frage nach Erscheinungsformen, Ursachen und Folgen von sozialer Differenzierung und Ungleichheit im sozialen Wandel auseinander. Sozialstrukturell steht die Frage im Vordergrund, wie aus bloßer Verschiedenartigkeit von Gesellschaftsmitgliedern wie Geschlecht, Alter, Ethnizität, soziale Herkunft - soziale Ungleichheiten im Hinblick auf Lebenschancen und -risiken werden. So wird im Rahmen des BA-Grundlagenmoduls immer wieder gefragt: Wer hat welche Bildungschancen? Wer wird was? Wer hat wie viel? Wer kommt nach oben? Wer bekommt welche Krankheit und wer stirbt wann? Wer trifft wen? Wer heiratet wen? Wer bekommt wann (noch) Kinder? Die Wahrnehmung und Bewertung von sozialen Ungleichheiten wird im Rahmen der Frage nach sozialen Bedingungen der Ausbildung spezifischer Sichtweisen auf Gerechtigkeit untersucht.

\section{Forschung und Lehre zur Sozialstruktur und sozialer Ungleichheit - ein überblick}

In den 1990er Jahren wurde die Forschung zu Sozialstruktur und sozialer Ungleichheit in einer neu gegründeten Sektion »Soziale Ungleichheit und Sozialstrukturanalyse« der Deutschen Gesellschaft für Soziologie institutionalisiert. An der Fakultät für Soziologie der Universität Bielefeld begann die Lehre und Forschung zur Sozialstruktur und sozialer Ungleichheit in Professuren für Allgemeine Soziologie in den 1980er Jahren. Prof. Dr. Hansjürgen Daheim (zum 01.03.1979 ernannt; Denomination: Allgemeine Soziologie) erinnert sich im Telefongespräch, wie er damals durch den Dekan, Prof. Dr. Berger, gefragt wurde, Seminare zur Sozialstrukturanalyse anzubieten. Er brachte ja diesbezüglich Erfahrungen aus seiner Zeit an der Universität Regensburg 
mit. An die lebhaften Diskussionen zu kleinen Aufsätzen im Seminar, z.B. zur De-Industrialisierung, und an die Vergabe einer Dissertation zur Völklinger Hütte erinnert Prof. Dr. Daheim sich noch gern. Prof. Dr. Elmar Lange wurde kurz nach Prof. Dr. Daheim zum 16.09.1982 mit der Denomination Soziologie, insbesondere Berufssoziologie, berufen und lehrte von da an die Einführungsveranstaltung in die Sozialstrukturanalyse mit ihm im Wechsel. In eingeworbenen Forschungsprojekten, z.B. bei der Deutschen Forschungsgemeinschaft oder dem Jugendamt der Stadt Bielefeld und in Projekten, die er mit Mitteln der Universität Bielefeld finanzierte, untersuchte Prof. Lange mit Methoden der quantitativen Querschnitts- und Längsschnitterhebung die Eltern- und Familienbildung in Bielefeld, den Übergang von der Hochschule in das Beschäftigungssystem, die Hochschulexpansion oder den Wandel des Jugendkonsums. Für die Bundesanstalt für Arbeit evaluierte er z.B. personelle Interventionsmaßnahmen auf Basis von Feldexperimenten. Mit Prof. Dr. rer. pol. Dr. h. c. Hans-Peter Blossfeld, Lehrstuhlinhaber für Allgemeine Soziologie, insbesondere Theorie und Empirie von Sozialstrukturen und Wirtschaftssystemen von 1998-2002, wurde die theorieorientierte empirische Forschung zur Sozialstruktur und sozialer Ungleichheit an der Universität Bielefeld weiter etabliert und gefestigt. Er warb in seiner Bielefelder Zeit das von der VW Stiftung geförderte Projekt »GlobalLife« (1999-2005) ein, in dem er mit zehn Mitarbeitern international vergleichend die Auswirkungen des Globalisierungsprozesses auf individuelle Lebens- und Erwerbsverläufe untersuchte. Prof. Blossfeld erläutert im persönlichen Telefongespräch, dass im Projekt von der Annahme ausgegangen wurde, dass durch Globalisierung sozialer Wandel beschleunigt wird, da auf Mikroebene Globalisierung für den Handelnden mit mehr Handlungsoptionen und mehr Unsicherheit einhergeht. Noch heute ist eine theorieorientierte quantitative empirische Forschung zur Sozialstruktur und sozialer Ungleichheit das Leitkonzept des Arbeitsbereiches, welches die derzeitigen Professoren, Prof. Dr. Martin Diewald seit 2004 (Denomination: Sozialstrukturanalyse) und Prof. Dr. Stefan Liebig seit 2008 (Denomination: soziale Ungleichheit und Sozialstrukturanalyse), sowie ihre Mitarbeiter_innen des Arbeitsbereiches vertreten. Soziale Ungleichheitsforschung war zudem von 2011 bis 2016 im SFB 882 »Von Heterogenitäten zu Ungleichheiten« an der Fakultät für Soziologie verbundförmig institutionalisiert. Eine Institutionalisierung der Forschung zu den Folgen der Digitalisierung der Arbeitswelt für soziale Ungleichheiten wurde zudem durch die Besetzung einer Juniorprofessur zu »Technischen und Sozialen Wandel« seit Februar 2018 (besetzt mit Anja-Kristin Abendroth) vorgenommen. 


\section{Zentrale Forschungsfragen im Arbeitsbereich Sozialstruktur und sozialer Ungleichheit}

Eine lange Tradition in Lehre und Forschung hat die Lebenslaufperspektive, die Interdependenzen von Lebensbereichen (Familie, Bildung, Arbeit) sowie die Prägung des Lebenslaufes durch zeitbezogene Ereignisse in den Blick nimmt und seit Prof. Blossfeld immer weiter ausgearbeitet wurde. In dem derzeitigen DFG-geförderten Projekt »LINOS « von Prof. Stefan Liebig werden strukturelle Bedingungen von Gerechtigkeitseinstellungen über den Lebensverlauf in den Blick genommen. Im Mittelpunkt stehen dabei Gerechtigkeitseinstellungen in Bezug auf das eigene Erwerbseinkommen und die Einkommensverteilung in der Gesellschaft. Des Weiteren geht es um die Bewertung von Regeln, nach denen Güter und Lasten in der Gesellschaft verteilt werden sollten und von Verfahren, wie Ungleichheiten in der Gesellschaft generiert werden. Mittlerweile ist die Lebenslaufperspektive am Arbeitsbereich zudem auch interdisziplinärer geworden. So wird im Rahmen der langfristig angelegten Zwillingsstudie »Twinlife« von Prof. Martin Diewald, Prof. Dr. Rainer Riemann (Psychologie) und Prof. Dr. Frank M. Spinath (Psychologie, Universität des Saarlandes) in einem interdisziplinären DFG-Langfristvorhaben gefragt, wie sich genetische und soziale Einflüsse auf die Lebenschancen, gesellschaftliche Position und soziale Mobilität von Menschen auswirken und welche vermittelnden Prozesse dabei eine Rolle spielen.

Seit dem Sonderforschungsbereich 882, „Von Heterogenitäten zu Ungleichheiten «, ist auch die organisationale Ungleichheitsperspektive zentraler Bestandteil des Arbeitsbereiches. Hier wird insbesondere auf Basis relationaler Ungleichheitstheorie (Tomaskovic-Devey 2014) gefragt, welche Rolle Organisationen bei der Allokation und Distribution unterschiedlicher Arten von sozialen Gütern und Positionen spielen, und insbesondere welchen Einfluss sie auf individuelle Lebenschancen haben. Bei der Analyse sozialer Einstellungen, Norm- und Wertorientierungen werden Organisationen zudem als spezifische soziale Kontexte der Einstellungsbildung berücksichtigt. Es wird also eine Erweiterung der klassischen Ansätze der Ungleichheits- und Einstellungsforschung durch eine organisationssoziologische Perspektive verfolgt. Beispielsweise untersucht das DFG-geförderte Projekt »Organisationale Ungleichheiten und Wechselwirkungen zwischen Verwirklichungschancen im Berufs- und Privatleben« (Abendroth, Diewald, Melzer), inwiefern sich betriebliche Strukturen, Kulturen und Politiken auf ungleiche Beschäftigungsbedingungen sowie Vereinbarkeitschancen von Beruf und Privatleben innerhalb von Betrieben auswirken, etwa im Vergleich der Geschlechter oder zwischen Personen ohne und mit Migrationshintergrund.

Eng verwoben mit den bereits erwähnten Perspektiven ist der Blick auf sozialen Wandel. Neue gesellschaftliche Entwicklungen wie Globalisierung, Individualisierung und zuletzt Digitalisierung wurden und werden durch den Arbeitsbereich aufgegriffen und gefragt, inwiefern damit Veränderungen in 
Lebenschancen und Ungleichheitsstrukturen verbunden sind. Globalisierung stand im erwähnten »GlobalLife« Projekt von Hans-Peter Blossfeld im Fokus. Aktuell geht der Arbeitsbereich durch die Beteiligung am interdisziplinären Forschungsschwerpunkt »Digitale Zukunft« und dem NRW Forschungskolleg: »Gestaltung von flexiblen Arbeitswelten«, gefördert durch das Ministerium für Kultur und Wissenschaft des Landes NRW, der Frage nach den Auswirkungen der Digitalisierung von Arbeit für Gratifikationen und Belastungen von verschiedenen Beschäftigtengruppen nach.

\section{Zentrale theoretische Zugänge im Arbeitsbereich Sozialstruktur und sozialer Ungleichheit}

Zwei zentrale theoretische Zugänge haben sich im Arbeitsbereich im Laufe der Zeit etabliert. Dies ist zum einen das Makro-Mikro-Makro Modell soziologischer Erklärung, welches auf den methodologischen Individualismus nach Coleman (1991) und Esser (1999) fußt und sich gegen eine starke Trennung von Makro- und Mikroperspektiven wendet. Zentrale Grundannahme ist die Einbettung von Akteuren in Opportunitätsstrukturen, die ihre Handlungen prägen. Die grundlegende Idee ist, dass Stabilität und Wandel gesellschaftlicher Ordnungen sich am besten durch Untersuchungen zum Einfluss kultureller, institutioneller und struktureller Rahmenbedingungen auf die Förderung und Forderung bestimmter Eigenschaften und Verhaltensweisen erfassen lassen. Zudem steht im Vordergrund, welche Folgen individuelles Entscheiden und Verhalten in Auseinandersetzung mit kulturellen, institutionellen und strukturellen Rahmenbedingungen nach sich zieht. Dem Modell zugrunde liegend wird gängiger Weise eine Handlungstheorie begrenzt rationaler Entscheidungen gewählt, in der auch Normen und Vertrauensprozesse Berücksichtigung finden, wie Prof. Blossfeld und Prof. Diewald im Gespräch erläutern. Prof. Diewald spezifiziert jedoch auch, dass dem klassischen methodologischen Individualismus eine recht einfache rationale Entscheidungstheorie zugrunde liegt. Mittlerweile werden Entscheidungen als Teil von durch psychische und biologische Bedingungen geprägte Routinen verstanden. Dies findet insbesondere in der bereits erwähnten interdisziplinären Lebenslaufperspektive Berücksichtigung.

Zum anderen hat seit dem Sonderforschungsbereich 882 »Von Heterogenitäten zu Ungleichheiten" der mechanistische Ansatz zur Erklärung sozialer Ungleichheiten (z.B. Diewald \& Faist 2011; Tomaskovic-Devey 2014) einen zentralen Stellenwert im Arbeitsbereich Sozialstruktur und soziale Ungleichheit. Ziel ist es, soziale Mechanismen zu identifizieren und zu systematisieren die aus Heterogenitäten wie Geschlecht, Migrationshintergrund, Bildung oder Alter soziale Ungleichheiten entstehen lassen. In der vielfach verwendeten Theorie relationaler Ungleichheiten (Tomaskovic-Devey 2014), wird der Forschungsstand zu Mechanismen der Ungleichheitsgenese in Organisationen 
zusammengefasst und weiter ausgearbeitet. Dabei spezifiziert die Theorie insbesondere die unterschiedliche Auswirkung von abstrakten Mechanismen der Ungleichheitsgenese (Chancenhortung, Ausbeutung, Claims Making und Resource Pooling) in Organisationen, da diese sich aufgrund unterschiedlicher Historien, Zusammensetzungen, Strukturen und Umwelten durch eigene Ungleichheitsregime auszeichnen.

\section{Zentrale Methodische Zugänge im Arbeitsbereich Sozialstruktur und soziale ungleichheit}

Der Arbeitsbereich verfolgt eine theorieorientierte empirische Forschung zur Sozialstruktur und sozialer Ungleichheit. Dabei orientiert sich der Arbeitsbereich an quantitativen Designs mit hohen Ansprüchen an Nachvollziehbarkeit und Überprüfbarkeit sowie damit einhergehender Archivierung und Dokumentation von Daten und Analysen als professionellen Gütekriterien. Methodisch wird versucht, sich kausalen Aussagen zu nähern durch Analyse von Längsschnittdaten und auch mittels methodischen Innovationen wie der Kontrolle von Selektion und unbeobachtbarer Heterogenität in verlinkten Betriebs- und Beschäftigtendaten mit Hilfe von Fixed-Effect-Organisationsanalysen. Dies soll jedoch nicht bedeuten, dass qualitativen Methoden die Daseinsberechtigung abgesprochen wird, erläutert Prof. Diewald. Quantitative Daten und Methoden sind zwar für die Beschreibung der Sozialstruktur Deutschlands oder anderer Länder unabdingbar. Nichtsdestotrotz werden neuere Entwicklungen von Mixed-Methods-Verfahren im Arbeitsbereich als sehr positiv bewertet.

\section{Lehre im Arbeitsbereich Sozialstruktur und soziale Ungleichheit}

Zu Beginn der Lehre zur Sozialstruktur und sozialer Ungleichheit an der Fakultät für Soziologie erfolgte die Einführungsveranstaltung im ersten Semester des Grundstudiums mit Schwerpunkten in den Themen soziale Ungleichheit, demografische Entwicklung, familiale und neue Lebensformen, soziale Schichtung und Lebenschancen. Um 2000 wurde die Veranstaltung in das dritte Semester nach der Ausbildung in quantitativen Methoden verlegt. Mit dem Übergang zum Bachelor- und Mastersystem ergänzten eine Übung mit Tutorium sowie ein Seminar die einführende Vorlesung zur Sozialstruktur und sozialer Ungleichheit. In der Vorlesung werden nach wie vor theoretisches und inhaltliches Grundwissen zur Sozialstruktur Deutschlands und ihre Besonderheiten im Vergleich zu anderen Gesellschaften bereitgestellt. Die begleitende Übung mit Tutorium vermittelt einen Überblick über Instrumente der Sozialstrukturanalyse, d.h. vor allem die großen Studien, die für entsprechen- 
de Analysen herangezogen werden können. Dazu werden in der Vorlesung kurz zuvor behandelte Konzepte und Zusammenhangsmuster mit Hilfe eines Statistik-Programmpakets auf Basis in der Übung vorgestellter Datensätze in einfacher Form praktisch umgesetzt. In dem Seminar wird die Möglichkeit geboten, am Beispiel ausgewählter Themen die Fähigkeit zu praktischer Sozialstrukturanalyse zu vertiefen (vgl. www.uni-bielefeld.de/soz/ab5/lehre.html).

Im Master werden im Modul Sozialstrukturanalyse und soziale Ungleichheit regelmäßig im Wintersemester Seminare angeboten, die eher allgemeineres Grundlagenwissen vermitteln. Im Sommersemester finden dagegen eher theoretisch oder empirisch vertiefende Seminare statt. Themenschwerpunkte sind soziale Ungleichheit, empirische Gerechtigkeitsforschung, Lebenslaufforschung, Organisationen und Ungleichheit, Familie und Partnerschaft, Sociogenomics, Soziale Mobilität, Arbeitsmarktforschung, Methoden der Sozialstrukturanalyse, Soziologische Theorie(Rational-Choice-Theorien, Relationale Ungleichheitstheorie, Mechanismen der Ungleichheitsgenese).

\section{Resümee und Ausblick}

Wo geht die Reise in den nächsten Jahren hin? Ein »weiter so « in theorieorientierter empirischer Forschung scheint klar und empfiehlt auch Prof. Blossfeld im Gespräch anlässlich des Geburtstages der Fakultät für die nächsten 50 Jahre. Auch inhaltlich wird die interdisziplinäre Lebensverlaufsperspektive und die Perspektive auf organisationale Ungleichheitsregime Forschung und Lehre im Arbeitsbereich weiter prägen. Prof. Blossfeld und Prof. Diewald betonen zudem beide, dass die Vielfalt und Breite des Faches der Soziologie, gebündelt an einer Fakultät, als Standortvorteil für den Arbeitsbereich wahrgenommen wird. Die Breite der Fakultät schafft im Vergleich zu einseitig strukturierten Fakultäten vielfältigere Möglichkeiten des Austausches. Dies stärker zu nutzen wünscht Martin Diewald dem Arbeitsbereich und der Fakultät für die Zukunft. Die themenzentrierte und interdisziplinäre Zusammenarbeit im Sonderforschungsbereich 882 von Gruppen, die unterschiedliche Methoden und Ansätze verfolgen, war hier ein erster Versuch. Für erfolgreiche Ergebnisse braucht es jedoch mehr Zeit als dem Sonderforschungsbereich bisher zugestanden wurde. Eine neue Strukturierung mit einer Überwindung von Fachgrenzen und themenzentriert orientierten Fakultäten bleibt damit ein wünschenswertes Zukunftsszenario, das noch in Angriff genommen werden sollte. 


\section{$\mathbf{L}_{\text {iteratur }}$}

Coleman, J.S. (1991): Grundlagen der Sozialtheorie. Band 1: Handlungen und Handlungssysteme. München: Oldenbourg.

Diewald, M. \& Faist, T. (2011): Von Heterogenitäten zu Ungleichheiten: Soziale Mechanismen als Erklärungsansatz der Genese sozialer Ungleichheiten. Berliner Journal für Soziologie 21(1): 91-114.

Esser, H. (1999): Soziologie. Spezielle Grundlagen. Band 1: Situationslogik und Handeln. Frankfurt a.M./New York: Campus.

Tomaskovic-Devey, D. (2014): The relational generation of workplace inequalities. Social Currents 1: 51-73. 